\title{
Analytical Model of Modified Traffic Control in an ATM Computer Network
}

The ABR class of ATM computer networks uses feedback information that is generated by net switches and destination and is sent back to a source of data to control the net load. A modification of the standard traffic management of the ATM network is presented, based on the idea of using RM cells to inform the source about congestion. An analytical model of a net switch is designed and mathematical relations are presented. The probability of queueing is considered. The analytical model of the switch was constructed for the purpose of analysing the network behaviour. It is used for investigating of cells passing through the network. We describe the model below. The submitted analytical results show that this method reduces congestion and improves throughput.

Keywords: Throughput, congestion control, modelling, RM cell, cell refusal.

\section{Introduction}

The ATM computer network consists of switches, sources and destinations of the transported data. The ATM network is based on switching packets of the same length called cells. Switches can transmit packets from their input to their output and also get information about the intensity of the traffic. Network traffic management is a significant process used for proper functioning of the network. The traffic management is based on a specific algorithm. To avoid congestion, current ATM networks use techniques of timeout, duplicate acknowledge or explicit congestion notification.

For monitoring the state of the network, special cells called RM (Resource Management) are used in the ATM network. RM cells inform data sources about the maximum rate of flow acceptable by the network. The load of the network varies in time, and interference of different loads in the same point can cause congestion. When this happens, the memories of the switches are overloaded and incoming cells have to be refused, i.e. removed. In this paper a new method is proposed for an earlier reaction to congestion.

An analytical model of a switch was constructed for an analysis of the network behaviour. This model is used for analysing traffic through the network. A description of the model is given in the sections below.

\subsection{Standard traffic management}

There are several classes of traffic in the ATM network. This paper considers the class called Available Bit Rate (ABR), used for transmissing files.

The ABR class of ATM computer networks uses feedback information on data transport from switches and destination to the source to control the load. The feedback mechanism is essential for good throughput of networks enabling an earlier reaction of sources when congestion of switches occurs.

The transported data are arranged in packets of cells. These packets have the same length, and a special cell called the RM cell precedes every packet. The traffic management is based on RM cells. RM cells pass from the source to the destination and collect information about the maximum rate of generated load which is acceptable by a dedicated path. After their acceptance by the destination they are sent back in order to bring the feedback information to the source. The rate of the traffic load is adjusted in the source according to the value received from the RM cell.

The flow of data cells together with RM cells between switches SW(i) and SW(i+1) is shown in Fig. 1. The RM cells are pictured as black rectangles, and the other cells as white rectangles. The packets of data cells with the RM cell number 8 are sent from the data source, i.e. forwards. Only RM cells 2 and 3 are sent backwards to bring feedback information from the destination.

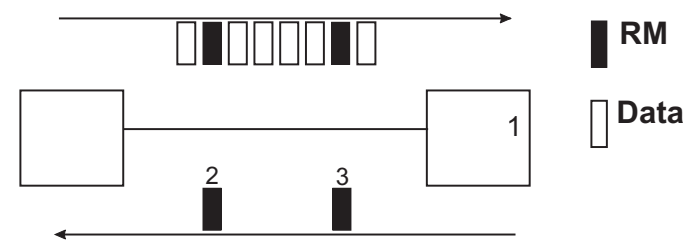

Fig. 1: The flow of counted RM cells

The disadvantage of this method is that the source of data obtains information about the current rate of the load, but it does not know which particular data was lost because it was refused within the switches. This information can be obtained later from the destination. In other words, RM cells always have to go along the whole path from the data source to the destination and back, see Fig. 5, 7.

\subsection{Proposed traffic model}

In the proposed model all RM cells are numbered by the source. The RM cells are returned by the switches at the moment of their congestion. If congestion occurs, the packets are refused. But associated RM cells are immediately sent back to inform the source of the loss of data. In this model, the source is informed earlier about the congestion in the network because RM cells did not pass along the whole path, see Fig. 5, 8 .

\section{Analytical model of the switch}

In this part the behaviour of basic network components switches - is studied. The behaviour of the switch can be described as a stationary queueing process. Let $(M|M| 1 \mid m)$ be a queueing process according to the Kendall notation. 
The symbols denote:

$M$ - distribution function of the cell interarrival time is exponential with the mean $\frac{1}{\lambda}, 0<\lambda<\infty$ and the arrival times are independent;

$M-$ distribution function of the cell service time is exponential with the mean $\frac{1}{\mu}, 0<\mu<\infty$ and the service times are independent;

1 - $\quad$ system with one place in service;

$m-\quad$ system of queue length $m$.

Notation used in the following text:

$\lambda$ - average arrival intensity;

$\mu-\quad$ average service rate;

$\rho=\lambda / \mu-$ traffic intensity.

Let us assume that cells coming to switch are stored in a switch queue of length $m$. Cells are refused if all of the $m$ waiting places are occupied, and further arriving cells are cancelled. The situation is shown in Fig. 2. The cell with index 0 is being transmitted and the following cells are waiting in the queue. The other incoming cells with an index equal to or greater than $m+1$ are refused.

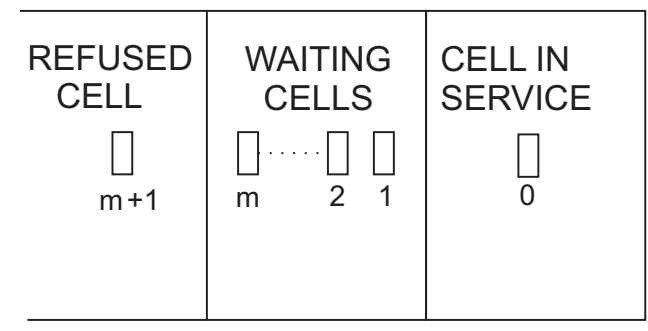

Fig. 2: The structure of a switch

The behaviour of this model is described by the probabilities of all possible queue lengths and by the cell waiting time in the queueing system.

The probability that there are just $k$ waiting cells in the queue is:

$$
\begin{aligned}
& p_{\mathrm{k}}=\frac{\rho^{\mathrm{k}}(1-\rho)}{1-\rho^{\mathrm{m}+2}} \text { for } \rho \neq 1 \text { and } \\
& p_{\mathrm{k}}=\frac{1}{m+2} \text { for } \rho=1, \quad 0 \leq k \leq m .
\end{aligned}
$$

The probability $p_{\mathrm{z}}$ of cell refusal (all places in the queue are occupied) is $p_{\mathrm{z}}=p_{\mathrm{m}+1}$ and its value equals:

$$
\begin{aligned}
& p_{\mathrm{Z}}=\frac{\rho^{\mathrm{m}+1}(1-\rho)}{1-\rho^{\mathrm{m}+2}} \text { for } \rho \neq 1 \text { and } \\
& p_{\mathrm{Z}}=\frac{1}{m+2} \text { for } \rho=1 .
\end{aligned}
$$

Fig. 3 shows the dependence of probability $p_{z}$ on the value $m$ for several values of traffic intensity $\rho$.

The cell waiting time $W$ in this queueing system is a random variable. For a description of the analytical model, the mean value $E(W)$ of the waiting time $W$ is used:

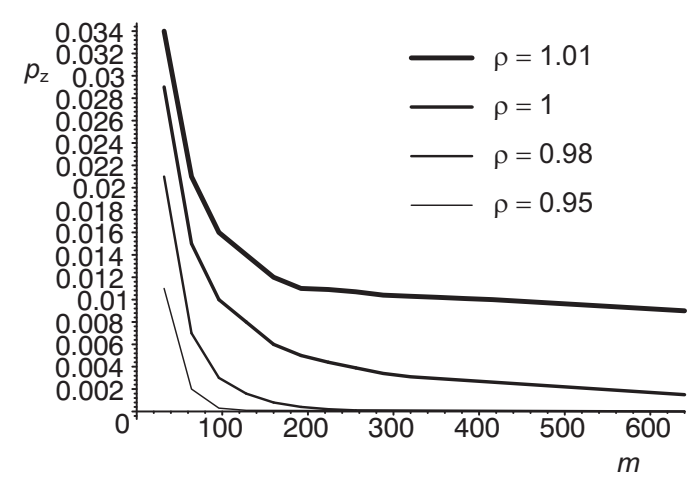

Fig. 3: Probability of cell refusal

$$
E(W)=\frac{p_{0}}{\mu} \sum_{k=1}^{m+1} k \rho^{\mathrm{k}} .
$$

Fig. 4 shows the dependence of the mean value $E(W)$ on the value $m$ for several values of traffic intensity $\rho$.

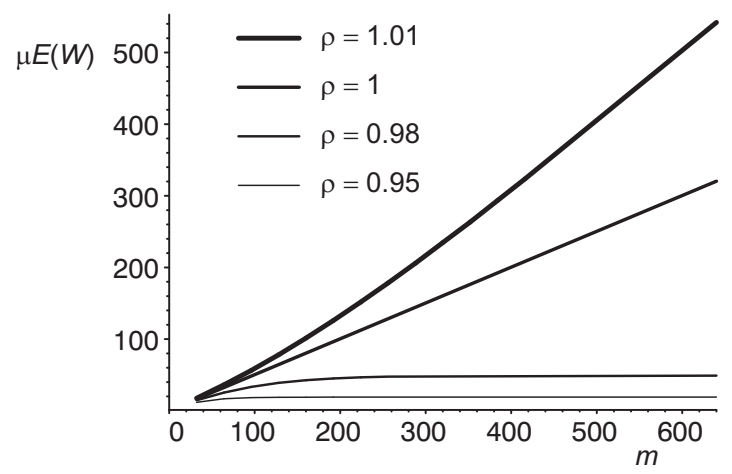

Fig. 4: Mean value of the waiting time in the queue

We can also use the probability of service $P_{\mathrm{p}}$,

$$
P_{\mathrm{p}}=1-p_{\mathrm{z}} \text {. }
$$

Other formulas describing the behavior of the queueing system can be found in [5].

The behavior of the system for values of traffic intensity $\rho$ close to 1 will be studied. In this case the refusal of cells occurs more frequently.

\section{Path of cell transport}

It is supposed that a path of cell transport from source $S$ to destination $D$ includes a sequence of $n$ switches $S W(i)$, $1 \leq i \leq n$. The same path is used for both forward transport of data cells together with RM cells and backward transport of RM cells. In the backward transport the RM cells pass through the switches in the reverse order. The switches are marked $S W(i), n+1 \leq i \leq 2 n$. Both paths are shown in Fig. 5 .

The following notation is used in Fig. 5:

$S$ - source of cells;

$D$ - destination of a transport;

$S W(i)-i$-th switch in the path;

$t_{\mathrm{i}}-\quad$ time period needed for cell transport along the line connecting switches $S W(i)$ and $S W(i+1)$. 


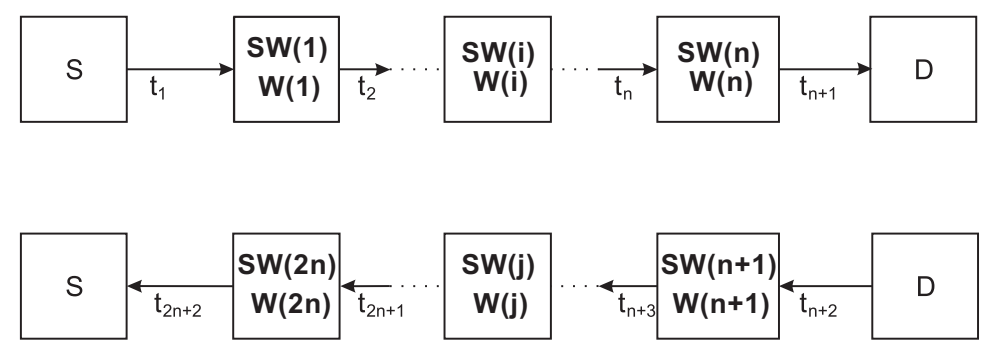

Fig. 5: Path of cell transport

\subsection{Characterization of cell transport}

The following cases of cell transport from the source to the destination were studied:

- The data cell is transported without being refused.

- The data cell is just once refused during the transport.

The formulas for transport time will be stated below, and we will use the weighted averages of the mean values of these time periods to compare the two models. We choose the probabilities of possible events as weight coefficients.

We do not consider the other cases of cell transport (when the number of refusals is greater than 1) because earlier studies $[1,2]$ have shown that the associated probabilities are negligible. The probabilities of cell refusal in a switch are shown in Fig. 3.

We use the following notation:

$p_{\mathrm{z}}(i)-$ probability of cell refusal in the $i$-th switch due to full switch buffer;

$P_{\mathrm{p}}(i)=1-\mathrm{p}_{\mathrm{z}}(i)-$ probability of service in the $i$-th switch;

$W(i)$ - waiting times in the queue of the $i$-th switch;

$E(W(i))$ - mean value of $W(i)$;

$t_{\mathrm{i}}$ - time period needed for cell transport along the line connecting switches $S W(i)$ and $S W(i+1)$.

The sample space of the considered events consists of independent elementary events $\{0,1,2, \ldots, n\}$. The symbols denote:

0 - $\quad$ the cell is transported without being refused in any switch;

$i$ - the cell is just once refused within the $i$-th switch $S W(i)$, $i=1,2, \ldots, n$.

We assume that the associated request (the RM cell transmitted from the destination to the source) and repeated sending of data cells are delivered without refusal.

The probabilities of the described events are conditional probabilities and their values can be approximated:

$$
\begin{aligned}
& P(0)=P_{\mathrm{p}}(1) P_{\mathrm{p}}(2) \ldots P_{\mathrm{p}}(n) ; \\
& P(i)=P_{\mathrm{p}}(1) \ldots P_{\mathrm{p}}(i-1) p_{\mathrm{z}}(i), i=1,2, \ldots, n .
\end{aligned}
$$

The values of $P_{\mathrm{p}}(i), i=1, \ldots, n$, are close to 1 and the values $p_{\mathrm{z}}(i) \ll 1, i=1, \ldots, n$. Therefore we can approximate the probabilities $P(i), i=0, \ldots, n$, as:

$$
\begin{aligned}
& P(0)=P^{0}=1-\left[p_{\mathrm{Z}}(1)+p_{\mathrm{Z}}(2)+\ldots+p_{\mathrm{Z}}(n)\right], \\
& P(i)=P^{1}(i)=p_{\mathrm{z}}(i), i=1, \ldots, n .
\end{aligned}
$$

We use the values $P^{0}, P^{1}(i), i=1, \ldots, n$ as the probabilities of the events $\{0,1, \ldots, n\}$.

We note that $P^{0}+\sum_{i=1}^{n} P^{1}(i)=1$.

We consider the individual cases of the cell transport denoted by the symbols $\{0,1, \ldots, n\}$ and we calculate their transport time.

We denote:

$T s_{\mathrm{t}} \quad$ total time period of cell transport in the standard model;

$T s_{\mathrm{t}}^{0} \quad$ total time period of cell transport without refusal in the standard model;

$T s_{\mathrm{t}}^{1}(i)$ total time period of cell transport with refusal in the $i$-th switch in the standard model;

$T p_{\mathrm{t}} \quad$ total time period of cell transport in the proposed model;

$T p_{\mathrm{t}}^{0} \quad$ total time period of cell transport without refusal in the proposed model;

$T p_{\mathrm{t}}^{1}(i)$ total time period of cell transport with refusal in the $i$-th switch in the proposed model.

\subsection{Cell transport without refusal}

The value $\mathrm{P}^{0}$ is the probability of cell transport without refusal within any switch of the path. It is actually the probability of a successful transmission without loss of any cell.

The influence of the proposed modification does not appear in this case and the transport times in the standard and proposed models are the same. In both models cells pass along the whole path from source $S$ to destination $D$. This path is shown in Fig. 6.

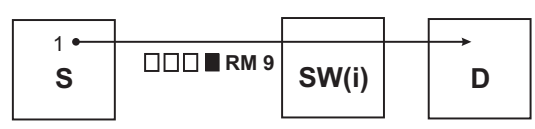

Fig. 6: Path of a cell transport without refusal

The total time periods $T s_{\mathrm{t}}^{0}$ and $T p_{\mathrm{t}}^{0}$ needed for the transport of a particular cell along the whole path in the standard and proposed models are equal, and are

$$
T s_{\mathrm{t}}^{0}=T p_{\mathrm{t}}^{0}=\sum_{k=1}^{n+1} t_{\mathrm{k}}+\sum_{k=1}^{n} W(k)
$$


The mean value of time $T s_{\mathrm{t}}(0)$ and $T p_{\mathrm{t}}(0)$ is

$$
E\left(T s_{\mathrm{t}}^{0}\right)=E\left(T p_{\mathrm{t}}^{0}\right)=\sum_{k=1}^{n+1} t_{\mathrm{k}}+\sum_{k=1}^{n} E(W(k))
$$

because only time periods $W(k)$ are random quantities.

\subsection{Cell transport with refusal of cells in the standard model}

We calculate the transport time $T s_{\mathrm{t}}(i)$. The assumption is that a cell is refused in the $i$-th switch $S W(i)$. The destination must call for repeated sending of the refused cell. The probability $P^{1}(i)=p_{\mathrm{Z}}(i)$ of this case is given in (5).

The path of a cell transport in the standard model is shown in Fig. 7. The switch $S W(i)$ is congested and that is why a cell is refused. Information about a cell being refused is not known before an uncompleted packet is received by destination $D$. Such a situation results in a request for a repeated transmission of data cells until the complete packet arrives at its destination.

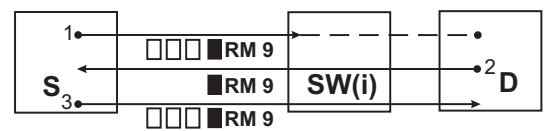

Fig. 7: Path of a cell transport with refusal in the standard model

The cell and the request have to pass the path three times and the total time period needed for the cell transport $T s_{\mathrm{t}}^{1}(i)$, $i=1, \ldots, n$, is

$T s_{t}^{1}(i)=2\left(\sum_{k=1}^{n+1} t_{\mathrm{k}}+\sum_{k=1}^{n} W(k)\right)+\sum_{k=n+2}^{2 n+2} t_{\mathrm{k}}+\sum_{k=n+1}^{2 n} W(k)$.

The mean value of the time period $E\left(T s_{\mathrm{t}}^{1}(i)\right)$ is

$$
\begin{aligned}
E\left(T s_{\mathrm{t}}^{1}(i)\right) & =2\left(\sum_{k=1}^{n+1} t_{\mathrm{k}}+\sum_{k=1}^{n} E(W(k))\right)+ \\
& +\sum_{k=n+2}^{2 n+2} t_{\mathrm{k}}+\sum_{k=n+1}^{2 n} E(W(k)) .
\end{aligned}
$$

\subsection{Cell transport with refusal of cells in the proposed model}

In the proposed modification, the cell transport path and the associated request are shown in Fig. 8. The request for the repeated sending is sent immediately from the congested switch $S W(i)$. We can see that in this case the cell and the associated request for its repeated sending does not very often pass along all the way from the source to the destination.

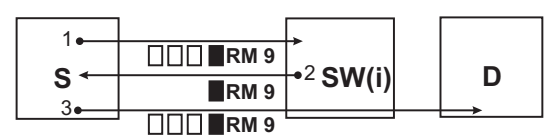

Fig. 8: Path of a cell transport with refusal in the proposed model
The cell is refused in the $i$-th switch with the probability $P^{1}(i)=p_{\mathrm{z}}(i)$.

The time periods needed for the transport along the individual parts of the whole path are:

1. $\quad \sum_{k=1}^{i} t_{\mathrm{k}}+\sum_{k=1}^{i-1} W(k)$

This is the time period of cell transport from source $S$ to switch $S W(i)$ :

2. $\sum_{k=2 n-i+3}^{2 n+2} t_{\mathrm{k}}+\sum_{k=2 n-i+2}^{2 n} W(k)$

This is the time period needed for transmission of the repeated sending request from switch $S W(i)$ to source $S$.

3. $\quad \sum_{k=1}^{n+1} t_{\mathrm{k}}+\sum_{k=1}^{n} W(k)$

This is the time period needed for transport of the cell from source $S$ to destination $D$.

The total time period $T p_{\mathrm{t}}^{1}(i)$ needed for the transport is:

$$
\begin{aligned}
T p_{\mathrm{t}}{ }^{1}(i)=\sum_{k=1}^{i} t_{\mathrm{k}} & +\sum_{k=1}^{i-1} W(k)+\sum_{k=2 n-i+3}^{2 n+2} t_{\mathrm{k}}+\sum_{k=2 n-i+2}^{2 n} W(k)+ \\
& +\sum_{k=1}^{n+1} t_{\mathrm{k}}+\sum_{k=1}^{n} W(k) .
\end{aligned}
$$

The mean value of time period $E\left(T p_{\mathrm{t}}^{1}(i)\right)$ is

$$
\begin{aligned}
E\left(T p_{\mathrm{t}}(i)\right) & =\sum_{k=1}^{i} t_{\mathrm{k}}+\sum_{k=1}^{i-1} E(W(k))+\sum_{k=2 n-i+3}^{2 n+2} t_{\mathrm{k}}+ \\
& +\sum_{k=2 n-i+2}^{2 n} E(W(k))+\sum_{k=1}^{n+1} t_{\mathrm{k}}+\sum_{k=1}^{n} E(W(k)) .
\end{aligned}
$$

\section{Comparison of cell transports}

We now compare the total time period of the cell transport in the standard model and in the proposed model. The transport time in the individual cases denoted by indeces $\{0,1, \ldots, n\}$ are random variables and the corresponding probabilities $P^{0}, P^{1}(i)$ are given in (5). As the mean value of the total transport time periods $T s_{\mathrm{t}}$ and $T p_{\mathrm{t}}$ we use the weighted averages of mean values $E\left(T s_{\mathrm{t}}^{0}\right), E\left(T s_{\mathrm{t}}^{1}(i)\right)$ and $E\left(T p_{\mathrm{t}}^{0}(i)\right), E\left(T p_{\mathrm{t}}^{1}(i)\right)$. The coefficients of weighted averages are the probabilities $P^{0}, P^{1}(i)$.

If we denote by $E\left(T s_{\mathrm{t}}\right)$ the mean value of the total time period of the cell transport in the standard model and by $E\left(T p_{\mathrm{t}}\right)$ the same quantity in the proposed model, we obtain:

$$
\begin{aligned}
& E\left(T s_{\mathrm{t}}\right)=E\left(T s_{\mathrm{t}}^{0}\right) P^{0}+\sum_{i=1}^{n} E\left(T s_{\mathrm{t}}^{1}(i)\right) P^{1}(i), \\
& E\left(T p_{\mathrm{t}}\right)=E\left(T p_{\mathrm{t}}^{0}\right) P^{0}+\sum_{i=1}^{n} E\left(T p_{\mathrm{t}}^{1}(i)\right) P^{1}(i) .
\end{aligned}
$$


The effect of the modification of the standard model is expressed by the difference

$$
\begin{aligned}
E\left(T s_{\mathrm{t}}\right)- & E\left(T p_{\mathrm{t}}\right)=\left[E\left(T s_{\mathrm{t}}^{0}\right)-E\left(T p_{\mathrm{t}}^{0}\right)\right] P^{0}+ \\
& +\sum_{i=1}^{n}\left[E\left(T s_{\mathrm{t}}^{1}(i)\right)-E\left(T p_{\mathrm{t}}^{1}(i)\right)\right] P^{1}(i)= \\
& =\sum_{i=1}^{n}\left[E\left(T s_{\mathrm{t}}^{1}(i)\right)-E\left(T p_{\mathrm{t}}^{1}(i)\right)\right] P^{1}(i)
\end{aligned}
$$

where we use the equality (7) $E\left(T s_{\mathrm{t}}^{0}\right)=E\left(T p_{\mathrm{t}}^{0}\right)$. The values $E\left(T s_{\mathrm{t}}^{1}(i)\right)$ and $E\left(T p_{\mathrm{t}}^{1}(i)\right)$ are given in (9), (11). The last expression depends on many parameters. We determine its value for one simple case. In the sequel we assume that the switches are identical, i.e. the waiting times $W(k)$ are the same and times $t_{\mathrm{k}}$ are equal.

We denote:

$$
\begin{aligned}
& \Delta t=t_{\mathrm{k}}, 1 \leq k \leq 2 n+2 ; \\
& E(W)=E(W(k)), 1 \leq k \leq 2 n ; \\
& p_{\mathrm{z}}=p_{\mathrm{z}}(k), P_{\mathrm{p}}=P_{\mathrm{p}}(k), 1 \leq k \leq 2 n .
\end{aligned}
$$

In this case we get

$$
\begin{aligned}
& E\left(T s_{\mathrm{t}}^{1}(i)\right)=[(3 n+3) \Delta t+3 n E(W)], \\
& E\left(T p_{\mathrm{t}}^{1}(i)\right)=[(n+2 i+1) \Delta t+(n+2 i-2) E(W)], \\
& i=1, \ldots, n \\
& E\left(T s_{\mathrm{t}}^{1}\right)-E\left(T p_{\mathrm{t}}^{1}\right)= \\
& =2 p_{\mathrm{z}} \sum_{i=1}^{n}[(n+1-i)(\Delta t+E(W))]= \\
& =n(n+1) p_{\mathrm{z}}(\Delta t+E(W)) .
\end{aligned}
$$

The value $E\left(T s_{\mathrm{t}}^{1}\right)-E\left(T p_{\mathrm{t}}^{1}\right)$ depends on the parameters of the switch $\left(E(W), p_{\mathrm{z}}\right)$ and the value $\Delta t$. We can suppose that the values $\Delta t \ll E(W)$ and therefore the comparison of the two models is demonstrated by the expression

$$
V=p_{\mathrm{z}} E(W) n(n+1) .
$$

The values $p_{\mathrm{z}}, E(W)$ and $P_{\mathrm{p}}$ are defined in (2), (3), and (4). The following table contains the values of $V$ for some path transport parameters.

Figs. 9 and 10 show the dependence of $V$ on the number $n$ of the switches for several values of traffic intensity $\rho$. Fig. 11 shows this dependence for several values of buffer storage $m$ and traffic intensity $\rho=1$.

The value of expression $V$ depends on the product of the probability $p_{\mathrm{z}}$ and the mean value of the waiting time $E(W)$. Figs. 12 and 13 show the dependence of the product $p_{\mathrm{z}} E(W)$ on value $m$ for several values of traffic intensity $\rho$.

\section{Conclusion}

The influence of the proposed modification of cell transport in an ATM computer network is tested. Analytical models of cell transport through standard and proposed modification are described. Formulas for the time period needed for cell transport for both models are presented.
Tab. 1: Influence of modification on the transport quality

\begin{tabular}{|c|c|c|c|c|c|}
\hline \multicolumn{2}{|c|}{} & \multicolumn{5}{|c|}{$V$} \\
\hline$m$ & $n$ & $\rho=1.01$ & $\rho=1$ & $\rho=0.99$ & $\rho=0.98$ \\
\hline 32 & 10 & 66.3 & 53.4 & 42.4 & 33.1 \\
\hline 32 & 20 & 253 & 204 & 162 & 126 \\
\hline 32 & 50 & 1536 & 1237 & 928 & 767 \\
\hline 64 & 10 & 81.4 & 53.7 & 33.9 & 18.8 \\
\hline 64 & 20 & 311 & 205 & 129 & 71.8 \\
\hline 64 & 50 & 1887 & 1244 & 785 & 436 \\
\hline 96 & 10 & 98.6 & 54.5 & 26.8 & 11.7 \\
\hline 96 & 20 & 376 & 208 & 102 & 44.8 \\
\hline 96 & 50 & 2285 & 1262 & 625 & 272 \\
\hline 128 & 10 & 120 & 54.7 & 21.2 & 6.8 \\
\hline 128 & 20 & 459 & 209 & 81 & 26 \\
\hline 128 & 50 & 2788 & 1267 & 492 & 158 \\
\hline
\end{tabular}

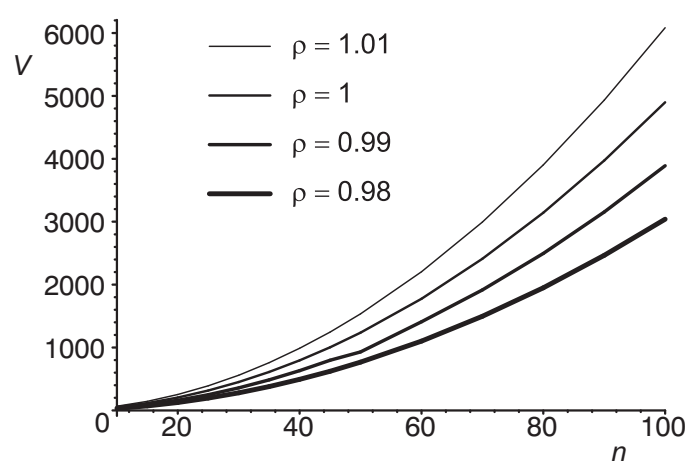

Fig. 9: Dependence of $V$ on parameter $n$ of the path

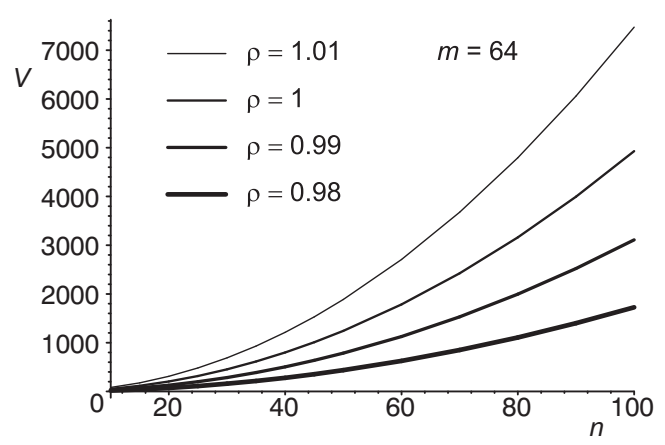

Fig. 10: Dependence of $V$ on parameter $n$ of the path

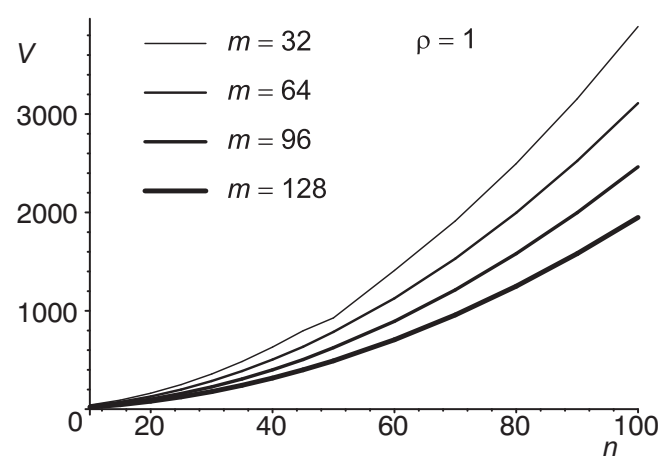

Fig. 11: Dependence of $V$ on parameter $n$ of the path 


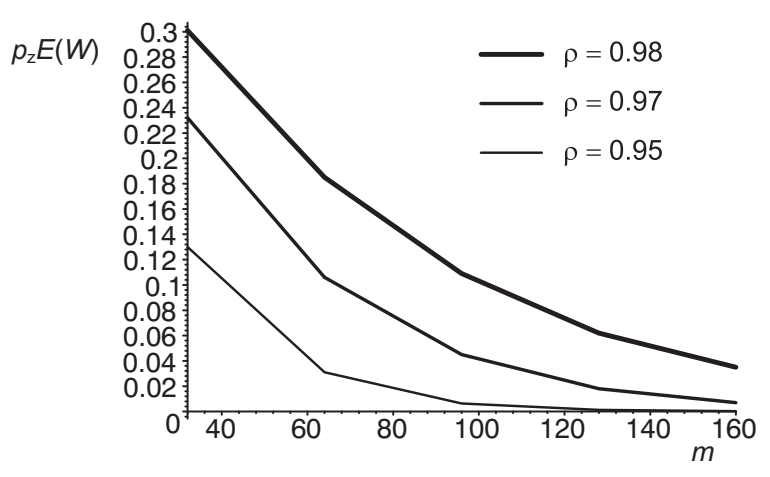

Fig. 12: Dependence of $p_{z} E(W)$ on value $m$

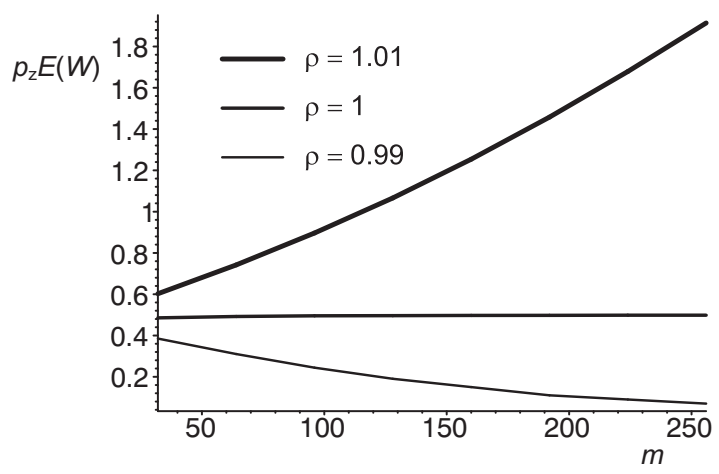

Fig. 13: Dependence of $p_{z} E(W)$ on value $m$

A comparison is made by the weighted averages of the mean values of the time period needed for cell transport $E\left(T s_{\mathrm{t}}\right)$, $E\left(T p_{\mathrm{t}}\right)$. As the coefficient of weighted values, we choose the probabilities of particular cases. We assumed transports without refusal and with one refusal only.

The differences of the mean values $E\left(T s_{\mathrm{t}}\right)$ and $E\left(T p_{\mathrm{t}}\right)(13)$ are approximated by the expression $V$. Its values in Table 1 and the graph dependences in Figs. 9, 10 and 11 show that the effect of the proposed modification grows with increased values of traffic intensity $\rho$. From Table1 and Fig.12, 13 it follows that if traffic intensity $\rho<1$, then the effect of the proposed modification decreases with increased values of buffer storage size $m$ while for $\rho \geq 1$ the effect of the proposed modification grows. This is caused by the fact that when $m$ increases, then the probability $p_{\mathrm{z}}$ of cell refusal decreases, but for $\rho \geq 1$ the mean of the waiting time $E(W)$ increases faster, hence the product $p_{\mathrm{z}} E(W)$ increases. If $\rho<1$ then this does not happen. We see from Tab. 1 that in this case the effect of the modification actually becomes smaller as we increase $m$.

\section{References}

[1] Filip, J.: Data transfer with modified RM cells. In: Proceedings of Workshop 97, Prague, CTU, 1997

[2] Filip, J.: Switching an alternative for high-speed networks. In: Proceedings of Workshop 96, Prague, CTU, 1996

[3] Filip, J.: Data Transports in ATM Networks. In: Proceedings of Workshop 2000, Prague, CTU, 2000

[4] Brandt, A., Fraenken, P., Lisek, B.: Stationary Stochastic Models. Akademie - Verlag Berlin, 1990

[5] Zitek, F.: Malé zastavení času. SPN Praha, 1975

[6] ATM Forum Technical Committee "Traffic management Specification Version 4.0”, April 1996

Ing. Jiří Filip

e-mail: fofis@post.cz

Ministerstvo zahraničních věcí České republiky Ministry of foreign affairs of the Czech republic Loretánské náměstí 5 ,

118 00, Praha 1, Czech Republic 